\section{USES OF ISOTOPES}

Les Radioéléments, Facteurs de Progrès Economique

Par Albert Nils. Pp. xii +214 . (Paris: Dunod, 1966.) 32 francs.

As an introduction the reader gets eight pages of very elementary explanations about the nucleus, the atom, artificial radioactivity and the production of artificially produced radioisotopes, written for a man with some basic knowledge of chemistry but not necessarily of physics.

The second chapter "Nuclear Energy and Radioisotopes" deals in a very succinct way with the present demand and resources of energy. The figures given in this chapter might be slightly out of date. The main part of the book is a summary of other publications on the advantages - mainly the financial ones-of using radioisotopes in industry, but also in medicine and agriculture.

It must be admitted that it is very difficult to undertake such a study in detail and therefore there are bound to be some shortcomings in such a book. Perhaps too great a simplification is applied to the economic treatment of isotopes in medicine and agriculture where, for example, entomological considerations such as the sterile male technique are treated in a few lines without emphasizing the enormous economic advantages of this technique. On the other hand, food preservation or extension of the shelf-life of food has been treated in far more detail. The main emphasis, however, is on the industrial uses of iso. topes and here some summaries and the main figures have been taken from the I.A.E.A. International Survey on the Use of Isotopes in Industry.

While the subject matter is surely of great interest to agriculturalists as well as to industrialists, the book is not written for them, or for the scientists or technicians. For the scientist it is perhaps too technical and for the technician it is not technical enough. It also has a bias to Belgian conditions.

I think that an industrialist, by quickly glancing through this book, might get quite a lot of first-hand information which might be useful to him although by no means comprehensive. A complete survey would be an extremely difficult, if not impossible, task.

Generally, it can be said that this book does not contribute much more, or in better form, than some of the publications referred to in its bibliography, but does contain some detailed information giving an idea of the field.

Henry Seligman

Introduction to Atomic and Nuclear Physics

By Rogers D. Rusk. Pp. xiv $+470+12$ plates. (London: Iliffe Books, Ltd.; New York: Appleton-Century-Crofts, 1965.) 63s, net.

ThIs book sets out to describe a large area of what is frequently called modern physics, aiming at providing an introduction for undergraduates. It treats a broad range of topics: quantum mechanics, relativity, atomic and molecular spectroscopy, nuclei, and glances at solid state physics and elementary particles. I think it is a failure, because the author is too frequently inaccurate, or expresses himself in a misleading way; in many cases these two possibilities are difficult to distinguish. As a reasonably typical example I quote from p. 323: "the suggestion by Heisenberg that the forces binding a proton in the nucleus to a neutron in the nucleus might arise from exchange of charge such that the proton momentarily becomes a neutron and the neutron momentarily becomes a proton and the two may scarcely be said to lead independent lives". Knowing something about this subject one can see some relation to the truth in these remarks but one cannot agree that they are correct: it is difficult to decide how much is actually wrong and how much is misleading presentation. Whichever it is, an undergraduate would surely be confused. There is a high density of such confusing statements, in places at least one to each page, so that it seems impossible to recommend the book. I also remark on another fault, which also occurs in other books. Each chapter is followed by a short list of books for "Recommended Reading". These range from popular accounts to advanced textbooks, many covering a much wider area than the particular chapter. The chief result of such general recommendations, without any guidance about what can be recommended in each book, must surely be to produce severe indigestion. I think this practice should be deplored.

A. B. ClegGa

\section{Introduction to the Theory of Flow Mechanics}

By Albert Betz. Translated by D. G. Randall. Pp. xvi +281 . (London and New York: Pergamon Press, 1966.) 75s. net.

THIs book is an edited translation of the German work of Betz, first published in 1959. The difficulty with textbooks on turbo-machine theory (and there are not many) is that they are either too elementary or they present a collection of mathematical solutions of various problems in the field. Lack of space usually prohibits a thorough description of these solutions so that much of the material is available only in the original papers. This book suffers slightly from this fault-although it can be read with immediate profit. A good collection of references is included, many of them to original work of many years standing and to much of the German work.

The contents are roughly as follows: Some twenty pages are devoted to general principles, a fairly good knowledge of fluid mechanics being assumed. The next fifty pages briefly discuss flow in ducts, including topics such as diffusers and boundary layers in curved ducts. This is followed by some seventy pages on cascade theory in which approximate treatments of ideal fluid problems are interleaved with discussions of the effects of losses, compressibility and end effects. The remainder of the book, apart from the appendixes, contains a discussion of almost every type of flow machine (including very short descriptions of thermodynamic machines, such as ramjets and pulse jets); the treatments are in many cases rather too brief. The appendixes refer mainly to the sections on cascade theory and include Betz's vortex influence charts and his cascade velocity and state diagrams. Perhaps the best part of the book is the section on cascade theory, although it is a pity that the translator could not have included an appendix on at least one of the theories outlined by Betz (in particular, the work of Isay). The translator is to be congratulated on achieving a most readable text.

J. W. RAILLY

\section{Problems of Atmospheric Circulation}

Edited by R. V. Garcia and T. F. Malone. (A Session of the Sixth International Space Science Symposium, Mar del Plata, Argentina, May 11-19, 1965.) Pp. vi + 186. (Washington, D.C.: Spartan Books; London: Macmillan and Co., Ltd., 1966.) 58s. net.

Aт a price which most research workers can afford for their private shelves, this book provides in one volume several review articles of the highest quality as well as much other material of great interest to all concerned with the broad problems of atmospheric physics.

There is a discussion on radiation and dynamics in the upper atmosphere from the vigorous laboratory at Leningrad under Professor K. Ya. Kondratiov. Professor R. E. Nowell (Massachusetts Institute of Technology) contributes a brilliant survey of the energy and momentum budget of the atmosphere above the tropopause. W. L. Webb combines and discusses the results of the 